\title{
Motion of a Swinging Atwood's Machine: Simulation and Analysis with Mathematica
}

\author{
Alexander N. Prokopenya
}

Received: 5 December 2016 / Revised: 20 February 2017 / Accepted: 21 February 2017 / Published online: 25 April 2017

(C) The Author(s) 2017. This article is an open access publication

\begin{abstract}
A generalized model of the Atwood machine when one body is constrained to move along a vertical axis while the other one can swing in a plane is considered. Combining symbolic and numerical calculations, we have obtained equations of motion of the system and analyzed their solutions. We have shown that oscillation can completely modify a motion of the system while the simple Atwood machine demonstrates only the uniformly accelerated motion of the bodies. The validity of the results obtained is demonstrated by means of the simulation of motion of swinging Atwood's machine with the computer algebra system Wolfram Mathematica.
\end{abstract}

Keywords Swinging Atwood's machine · Oscillation · Quasi-periodic motion · Simulation · Wolfram Mathematica

Mathematics Subject Classification Primary 34A34; Secondary 34C60

\section{Introduction}

An Atwood machine is a well-known device that is usually used in the course in physics to demonstrate the uniformly accelerated motion of a system (see [1]). It consists of two bodies, having masses $m_{1}$ and $m_{2}\left(m_{2}>m_{1}\right)$, attached to opposite ends of a massless inextensible thread wound round a massless, frictionless pulley. It is assumed that each body can move only along a vertical, and the thread doesn't slip on the pulley. Such Atwood's machine is a simple mechanical system with one degree freedom and, using Newton's second law, one can easily obtain its equation of motion and show that acceleration of each body is given by

$a=\frac{m_{2}-m_{1}}{m_{2}+m_{1}} g$

where $g$ is a gravity acceleration.

Obviously, any real Atwood's machine is much more complicated in comparison with the simplest device described above because there are at least two significant features of the system which have been neglected for simplicity. First, a pulley has some mass and, therefore, its nonzero moment of inertia about axis of rotation may

A. N. Prokopenya ( $\square)$

Warsaw University of Life Sciences - SGGW, Nowoursynowska Str. 159, 02-776 Warsaw, Poland

e-mail: alexander_prokopenya@sggw.pl 
influence noticeably on motion of the system. Second, in addition to translational motion, the bodies being hung on a flexible thread, can oscillate under an influence of the force of gravity. Therefore, real system has more than one degree of freedom and its behaviour is described by a complex system of differential equations whose general solution can not be found in symbolic form. Nevertheless, such equations can be solved numerically and influence of the mass of the pulley and oscillations of the bodies on system's motion can be investigated.

It should be noted that swinging Atwood's machine has been a subject of a number of papers (see [2-8]) and its mechanical behaviour has been studied quite well. In particular, it has been proven that the system of differential equations describing dynamics of swinging Atwood's machine is not integrable, in general. It has been shown also that, depending on the mass ratio $m_{2} / m_{1}$, the system can demonstrate different types of motion, namely, periodic, quasi-periodic, or chaotic motion but physical reasons of such behaviour of the system and significance of oscillation is not usually discussed.

The main purpose of the present paper is to analyze the terms appearing in the equations of motion owing to oscillations, and to demonstrate their crucial role for the system behaviour. And the computer algebra system system Wolfram Mathematica (see [9]) turns out to be a very good tool to do all necessary symbolic and numerical calculations and to visualize the results.

\section{Equations of Motion}

Let us consider the simplest generalization of Atwood's machine when moment of inertia of the pulley about axis of rotation is taken into account, and the body of mass $m_{1}$ is allowed to swing in a plane while the other body of mass $m_{2}$ is constrained to move only along vertical axis $O y$ (see Fig. 1). The corresponding system has two degrees of freedom and its geometrical configuration can be described in terms of two variables, namely, an angle $\varphi$ determining deviation of the thread from a vertical, and a length $r$ of the thread between the body $m_{1}$ and the point, where the thread departs from the pulley in case of $\varphi=0$. Then Cartesian coordinates $x_{1}, y_{1}, y_{2}$ of the bodies can be determined as

$$
\begin{aligned}
& x_{1}(t)=R \cos \varphi(t)+(r(t)+R \varphi(t)) \sin \varphi(t), \\
& y_{1}(t)=R \sin \varphi(t)-(r(t)+R \varphi(t)) \cos \varphi(t), \\
& y_{2}(t)=r(t)-L+\pi R,
\end{aligned}
$$

where $L$ is a length of the thread, $R$ is a radius of the pulley, and we have taken into account that the thread is a tangent to the pulley.

Doing standard symbolic calculation, we can write the Lagrangian function of the system as (see, for example, [10])

$$
\begin{aligned}
\mathcal{L}=\frac{1}{2} & \left(m_{1}+m_{2}+\frac{I_{0}}{R^{2}}\right) \dot{r}^{2}+\frac{m_{1}}{2}(r+R \varphi)^{2} \dot{\varphi}^{2} \\
& -m_{1} g(R \sin \varphi-(r+R \varphi) \cos \varphi)-m_{2} g r,
\end{aligned}
$$

where $I_{0}$ is a moment of inertia of the pulley, and the dot in $\dot{r}$ denotes the time derivative of the function $r(t)$. It is assumed also that the thread doesn't slip on the pulley and angular velocity of the pulley is completely determined by the velocity $\dot{y}_{2}$ of the body $m_{2}$.

Using the Lagrangian (2.1), we obtain the equations of motion of the system in the form

$$
\begin{aligned}
& \kappa \ddot{r}=-g(\mu-\cos \varphi)+(r+R \varphi) \dot{\varphi}^{2}, \\
& (r+R \varphi) \ddot{\varphi}=-g \sin \varphi-2 \dot{r} \dot{\varphi}-R \dot{\varphi}^{2},
\end{aligned}
$$

where

$$
\kappa=\frac{I_{0}+\left(m_{1}+m_{2}\right) R^{2}}{m_{1} R^{2}}>1, \quad \mu=\frac{m_{2}}{m_{1}} .
$$


Fig. 1 Swinging Atwood's machine

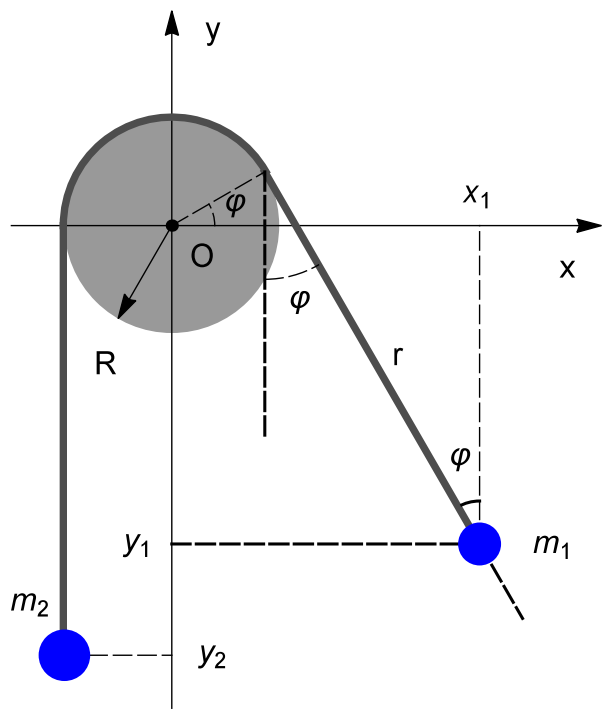

Note that in the absence of oscillations when $\varphi \equiv 0$, Eq. (2.3) is satisfied identically while Eq. (2.2) is easily solved and its solution is given by

$r(t)=r_{0}+v_{0} t-\frac{g(\mu-1) t^{2}}{2 \kappa}$,

where $r_{0}=r(0)$ and $v_{0}=\dot{r}(0)$ are initial distance and velocity, respectively. Obviously, in case of $\mu \neq 1$ solution (2.4) determines a uniformly accelerated motion of the bodies, and Atwood's machine was devised just to demonstrate this type of motion.

One can readily see from (2.4) that nonzero moment of inertia of the pulley results in decreasing the acceleration

$\ddot{r}=-\frac{g(\mu-1)}{\kappa}=-\frac{g\left(m_{2}-m_{1}\right)}{I_{0} / R^{2}+m_{1}+m_{2}}$,

in comparison with the case of massless pulley (see (1.1)). Therefore, taking into account a mass of the pulley in Atwood's machine without oscillations makes the system more inertial but doesn't change its behaviour in a qualitative sense.

\section{Motion of Swinging Atwood's Machine}

Taking into account planar oscillation of the body $m_{1}$ complicates the equations of motion significantly (see (2.2), (2.3)) and their general solution cannot be found in symbolic form. However, choosing some realistic values of the system parameters, we can obtain the corresponding numerical solution for different initial conditions and analyze motion of the system.

\subsection{Equal Masses}

In case of equal masses $(\mu=1)$ the bodies in the simple Atwood's machine can either move uniformly or rest, depending on the initial conditions. Let us consider the case when the system rests and we impart a small horizontal initial velocity to the body of mass $m_{1}$. As both bodies have the same mass and are initially in equilibrium, it seems 

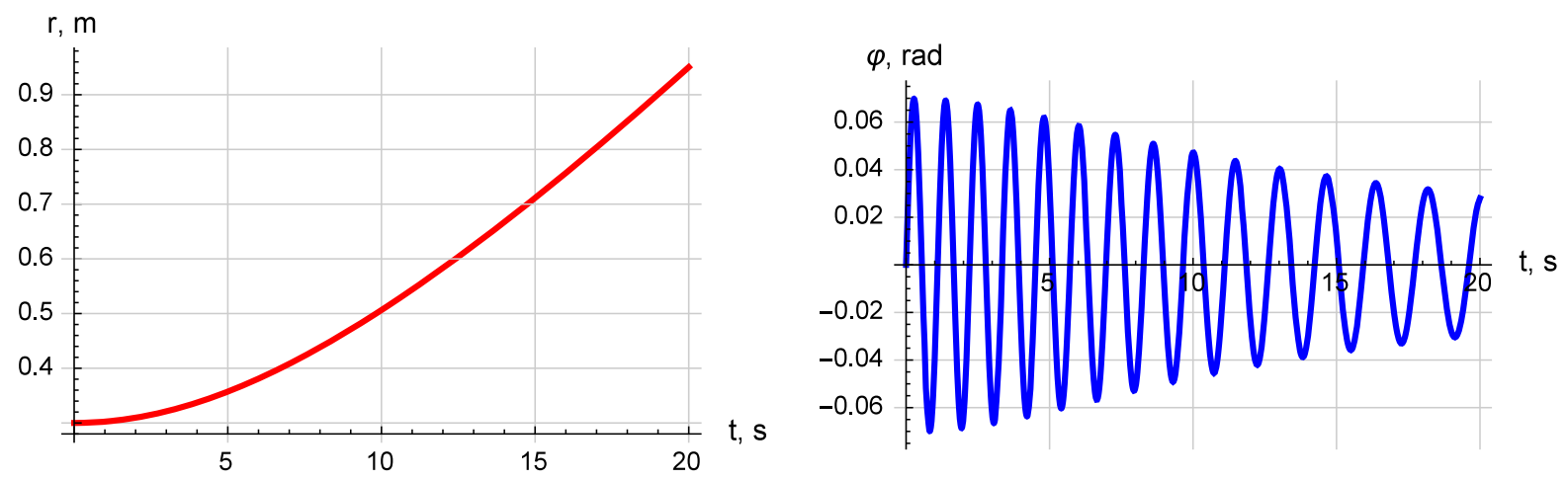

Fig. 2 Motion of the system in case of equal masses and $\kappa=2.5$
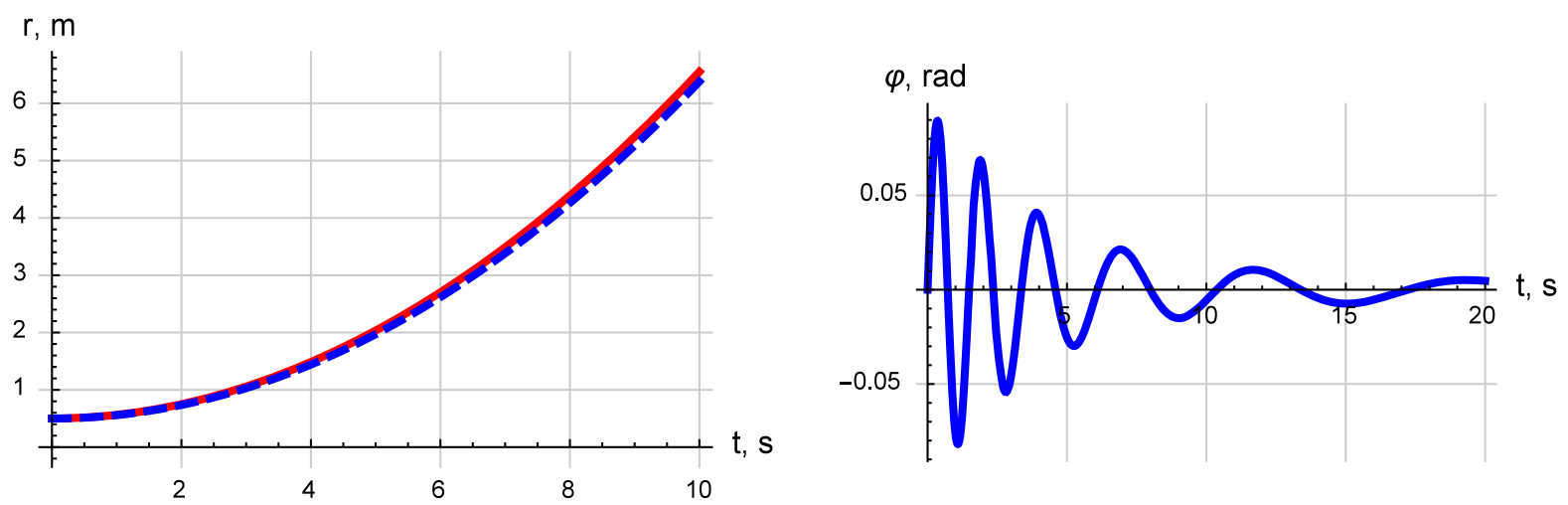

Fig. 3 Motion of the system in case of $\mu=0.97, \kappa=2.5$

to be quite natural to assume that the system will move in the neighbourhood of its equilibrium position. However, using the Mathematica built-in function NDSolve (see [9]) and obtaining numerical solution of the equations of motion (2.2), (2.3), we observe completely different behaviour of the system (see Fig. 2). It turns out that small oscillation of the body of mass $m_{1}$ causes an unbounded growth of the function $r(t)$ while amplitude of oscillation of the function $\varphi(t)$ decreases with time. It would be nothing strange if initial velocity of the body $m_{1}$ had a vertical component but is was directed horizontally.

\subsection{Unequal Masses $(\mu \neq 1)$}

Now let us consider the case when one body has a little bit greater mass than another one. Just such a case is usually realized experimentally when the corresponding demonstration is shown at lectures in physics.

In case of oscillation of the body having greater mass $(\mu<1)$ qualitative behaviour of the system doesn't change too much in comparison to the simple Atwood machine, the corresponding solutions $r(t)$ and $\varphi(t)$ of the system (2.2), (2.3) are shown in Fig. 3, where a dashed curve visualizes the function (2.4) for $v_{0}=0$. As we could expect the more massive body $m_{1}$ moves down and the function $r(t)$ deviates only a little bit from the solution (2.4). An amplitude of its oscillation decreases as the function $r(t)$ grows up similarly to the previous case of equal masses of the bodies.

In case of $m_{1}<m_{2}(\mu>1)$ and zero initial velocity of the bodies simple Atwood's machine demonstrates a motion in opposite direction and the function $r(t)$ decreases with time (see (2.4)). If we impart a small horizontal initial velocity to the body of smaller mass $m_{1}$ an initial stage of motion of the swinging Atwood machine looks similarly (see Fig. 4). But later behaviour of the swinging Atwood machine becomes completely different. The 

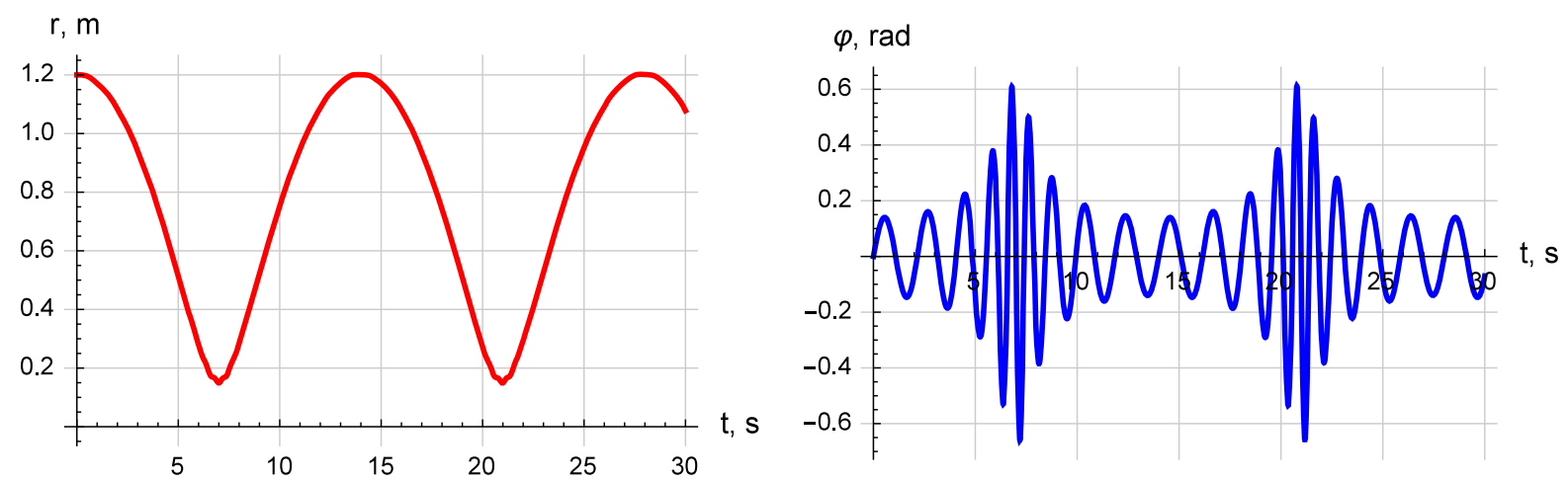

Fig. 4 Motion of the system in case of $\mu=1.02, \kappa=2.5$

Fig. 5 Swinging Atwood machine with two small pulleys

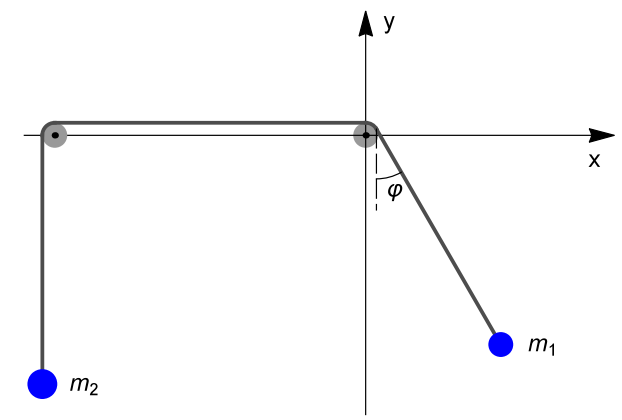

function $r(t)$ decreases first but after reaching some minimum it starts to increase what is not possible in case of the simple Atwood machine. One can readily see that decrease of the function $r(t)$ is accompanied by increase of an amplitude and frequency of the body $m_{1}$ oscillation while they decrease again when the system starts to move in opposite direction. As a result we observe a quasi-periodic motion of the system and it would be very difficult to predict such strange its behaviour without solving the equations of motion.

One can easily check also that for large enough difference of masses $m_{1}$ and $m_{2}\left(m_{1}<m_{2}\right)$ quasi-periodic motion of the system disappears and the function $r(t)$ decreases with time until the body $m_{1}$ reaches the pulley. But the question remains why oscillation of the body $m_{1}$ can modify the system motion so dramatically in case of small difference of masses of the bodies?

\section{Influence of Oscillation on the System Motion}

Obviously, the body of mass $m_{1}$ being hung on a flexible thread (see Fig. 1) may be treated as a pendulum of variable length $(r(t)+R \varphi(t))$. Numerical solutions of the equations of motion (see Figs. 2, 3, 4, where $R=0.05$ $\mathrm{m})$ show that the condition $|R \varphi(t)| \ll r(t)$ is fulfilled in all the considered cases. Therefore, one can assume that a change of the pendulum length $R \varphi(t)$ being a result of the thread winding on the pulley during oscillation doesn't influence on the system motion essentially and may be neglected. From the other side, replacing the pulley of finite radius by two separated small pulleys (see Fig. 5), one can obtain the Atwood machine, where the pendulum length changes only due to rotation of the pulleys. Equations of motion of such machine are obtained from Eqs. (2.2), (2.3) by substitution $R \rightarrow 0$ and are given by

$$
\begin{aligned}
\kappa \ddot{r} & =-g(\mu-\cos \varphi)+r \dot{\varphi}^{2}, \\
\ddot{\varphi} & =-\frac{g}{r} \sin \varphi-2 \frac{\dot{r}}{r} \dot{\varphi} .
\end{aligned}
$$


Note that numerical solutions of these equations differ only slightly from the solutions shown in Figs. 2, 3 and 4 while qualitative behaviour of the system remains the same. It means that oscillation of the body $m_{1}$ is a main factor determining dynamical behaviour of the system. And to clarify a role of oscillation it makes sense to investigate the simplified system (4.1), (4.2) first.

To explain a possibility for the body of smaller mass $m_{1}$ to move down and to pull the body of larger mass $m_{2}$ let us consider an equation of motion of a mathematical pendulum of length $r$, it is well-known and reads as (see, for example, [10])

$\ddot{\varphi}=-\frac{g}{r} \sin \varphi$.

Integrating this equation gives

$\dot{\varphi}^{2}=\frac{2 g}{r}\left(\cos \varphi-\cos \varphi_{0}\right)$,

where $\varphi_{0}$ is an amplitude of oscillation.

Using Newton's second law, one can easily obtain an expression for the thread tension

$F_{\text {ten }}=m_{1}\left(g \cos \varphi+r \dot{\varphi}^{2}\right)$.

Comparing Eqs. (4.1) and (4.5) shows that in fact the bodies acceleration $\ddot{r}$ is determined by a difference of the gravity force $m_{2} g$ and the thread tension $F_{\text {ten }}$ given by (4.5). Parameter $\kappa$ takes into account moments of inertia of the pulleys and decreases the acceleration $\ddot{r}$ but it doesn't influence on its sign. In absence of oscillation $(\varphi \equiv 0)$ the tension $F_{\text {ten }}$ reduces to the gravity force $m_{1} g$ and, therefore, the acceleration $\ddot{r}$ should be negative in case of $\mu>1$ or $m_{2}>m_{1}$. During oscillation the first term $m_{1} g \cos \varphi$ in the right-hand side of (4.5) becomes even smaller than the gravity force $m_{1} g$ but the centrifugal force $m_{1} r \dot{\varphi}^{2}$ arises and due to this the thread tension may become even greater than the gravity force $m_{1} g$.

Indeed, taking into account (4.4), we can rewrite Eq. (4.5) in the form

$F_{\text {ten }}=m_{1} g\left(3 \cos \varphi-2 \cos \varphi_{0}\right)$.

It means that the tension is an oscillating function of the angle $\varphi$ and its values belong to the interval

$m_{1} g \cos \varphi_{0} \leq F_{t e n} \leq m_{1} g\left(1+4 \sin ^{2} \frac{\varphi_{0}}{2}\right)$.

Therefore, its average value may exceed the gravity force $m_{1} g$ even for small amplitude $\varphi_{0}$.

Actually, in case of $\varphi_{0} \ll 1$ when $\sin \varphi \approx \varphi$ Eq. (4.3) describes harmonic oscillation of the pendulum. Expanding the cosine function in power series in terms of $\varphi$ up to the second order, we can rewrite Eq. (4.6) in the form

$F_{\text {ten }}=m_{1} g\left(1-\frac{3}{2} \varphi^{2}+\varphi_{0}^{2}\right)$

Then averaging the tension $F_{\text {ten }}$ over the period of oscillation $T=2 \pi \sqrt{r / g}$ gives

$F_{\text {ten }}^{a v}=\frac{1}{T} \int_{0}^{T} F_{\text {ten }} d t=\frac{m_{1} g}{\pi} \int_{-\varphi_{0}}^{\varphi_{0}}\left(1-\frac{3}{2} \varphi^{2}+\varphi_{0}^{2}\right) \frac{d \varphi}{\sqrt{\varphi_{0}^{2}-\varphi^{2}}}=m_{1} g\left(1+\frac{\varphi_{0}^{2}}{4}\right)$, 

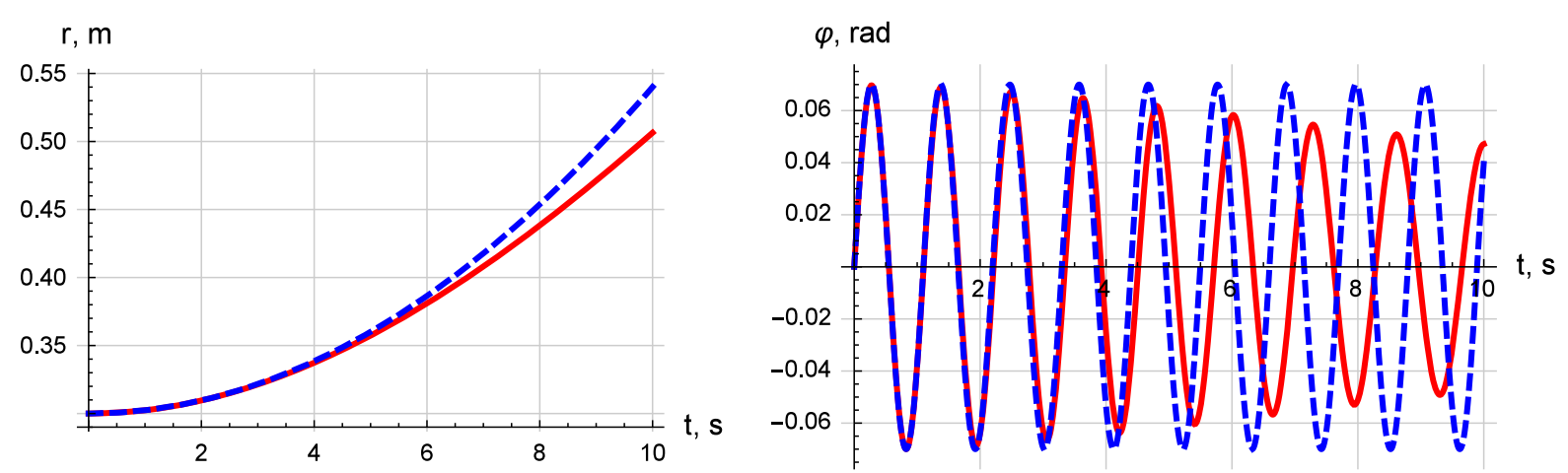

Fig. 6 Approximate solution (dashed blue curves) and numerical solution of the equations of motion (4.1), (4.2) (solid red curves) in case of $\mu=1, \kappa=2.5$ (colour figure online)

where Eq. (4.4) has been taken into account. Thus, the averaged tension $F_{t e n}^{a v}$ is greater than the gravity force $m_{1} g$ even for small amplitude of oscillation and it increases as the amplitude grows up. For larger amplitude $\varphi_{0}$ the calculation of $F_{t e n}^{a v}$ becomes more complicated but a final result $F_{t e n}^{a v}>m_{1} g$ remains valid.

Now it is quite evident that in case of equal masses of the bodies $(\mu=1)$ the oscillating body should move down and pull up the second body. Actually, for small amplitude of oscillation the sine function in (4.2) may be replaced by the corresponding linear term: $\sin \varphi \approx \varphi$. Besides, it follows from Fig. 2 that the ratio $\dot{r} / r$ remains small during the system motion and so the second term in the right-hand side of Eq. (4.2) may be neglected. As a result this equation reduces in zero approximation to the equation of harmonic oscillation and its solution can be written as

$\varphi(t)=\dot{\varphi}_{0} \sqrt{\frac{g}{r_{0}}} \sin \left(\sqrt{\frac{g}{r_{0}}} t\right)$,

where $\dot{\varphi}_{0}$ is an initial angular velocity of the body $m_{1}$, while its initial coordinates are $\varphi(0)=0, r(0)=r_{0}$. Comparing the approximate solution (4.8) with the corresponding numerical solution of the system (4.1)-(4.2) shows (see Fig. 6) that during the first two periods of oscillations both solutions are very close to each other.

Replacing the cosine function in (4.1) by its power series expansion in terms of $\varphi$ up to the second order and substituting expression (4.8), we can rewrite Eq. (4.1) in the form

$\ddot{r}=-\frac{g(\mu-1)}{\kappa}+\frac{r_{0} \dot{\varphi}_{0}^{2}}{4 \kappa}+\frac{3 r_{0} \dot{\varphi}_{0}^{2}}{4 \kappa} \cos \left(2 \sqrt{\frac{g}{r_{0}}} t\right)$

where again the function $r(t)$ in the right-hand side of (4.1) was replaced by its initial value $r_{0}$. Differential equation (4.9) can be easily solved and the corresponding solution is given by

$r(t)=r_{0}+\left(-\frac{g(\mu-1)}{\kappa}+\frac{r_{0} \dot{\varphi}_{0}^{2}}{4 \kappa}\right) \frac{t^{2}}{2}+\frac{3 r_{0}^{2} \dot{\varphi}_{0}^{2}}{16 g \kappa}\left(1-\cos \left(2 \sqrt{\frac{g}{r_{0}}} t\right)\right)$,

where initial conditions $\dot{r}(0)=0, r(0)=r_{0}$ have been taken into account. Visualization of this solution in case of equal masses of the bodies ( $\mu=1$ ) shows (see Fig. 6) that it describes correctly the first stage of the system motion. However, due to increase of the velocity $\dot{r}(t)$ with time the second term $2 \dot{r} \dot{\varphi} / r$ in the right-hand side of Eq. (4.2) becomes essential and its action may be treated as damping. As a result the amplitude of oscillation decreases (red curve in the right-hand side of Fig. 6). Besides, an increase of the pendulum length $r$ results in increase of the period of oscillation. Thus, one has to take into account the higher order terms in the power series expansion of the sine and cosine functions in Eqs. (4.1), (4.2) to obtain more accurate symbolic solution of the equations of motion. 

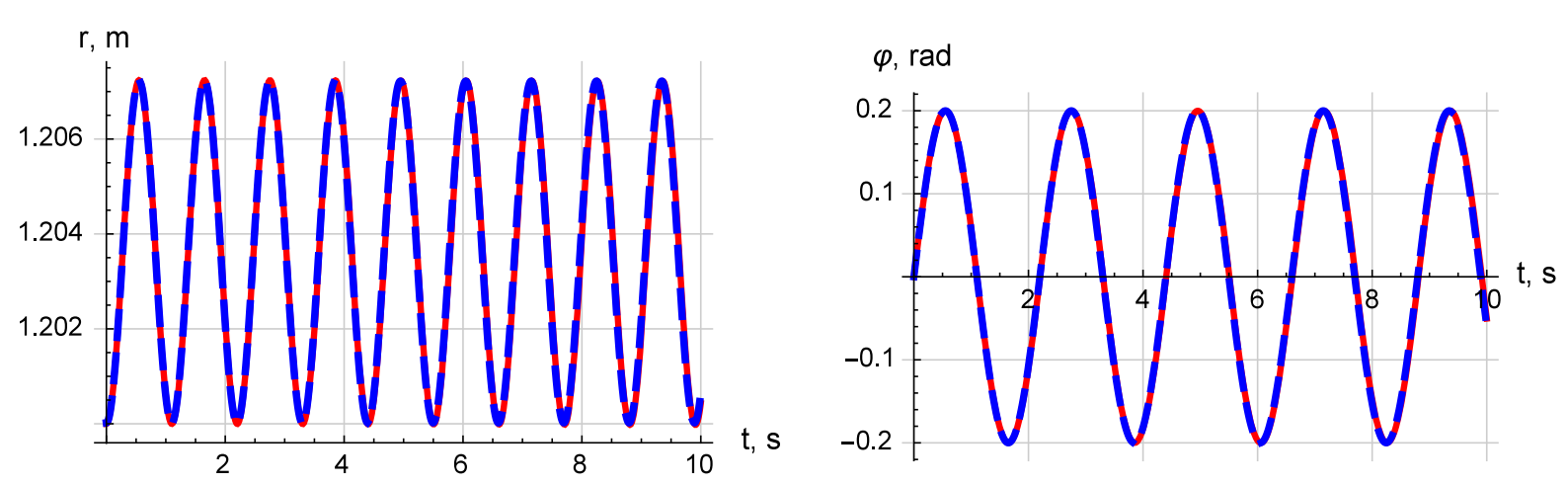

Fig. 7 Harmonic oscillations of the body $m_{1}$ near equilibrium, $\mu=1.01, \kappa=2.5$
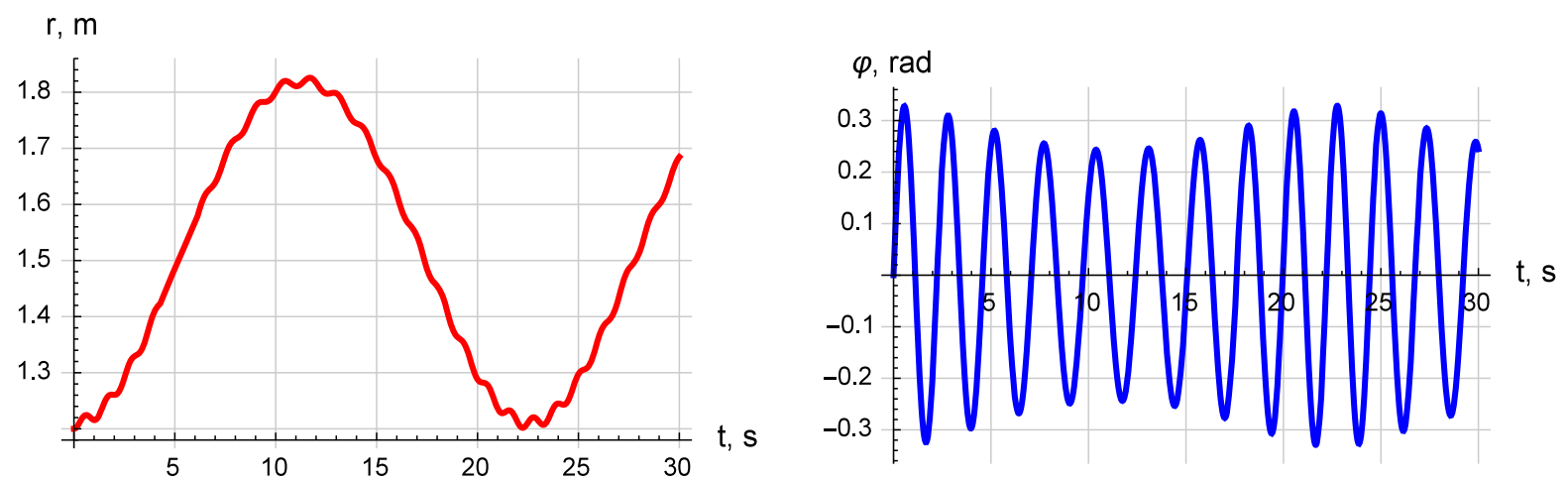

Fig. 8 Motion of the Atwood machine in case of $\varphi_{0}>\sqrt{4(\mu-1)}, \mu=1.02, \kappa=2.5$

Nevertheless, solutions (4.8), (4.10) give correct description of the first stage of the system motion in case of equal masses of the bodies.

Note that increasing the mass $m_{2}$ of the second body (or parameter $\mu$ ), one can reach a state of equilibrium when the averaged tension balances the gravity force $m_{2} g$ and the coefficient of $t^{2}$ in Eq. (4.10) becomes equal to zero. The corresponding value of parameter $\mu$ and the amplitude $\varphi_{0}$ are connected by the relationship $\varphi_{0}=\sqrt{4(\mu-1)}$. Of course, it should be a state of dynamical equilibrium because both functions $\varphi(t)$ and $r(t)$ determined by Eqs. (4.8) and (4.10), respectively, oscillate near equilibriums. It should be emphasized that comparing these functions (blue dashed curves in Fig. 7) with the corresponding numerical solutions of Eqs. (4.1), (4.2) (solid red curves in Fig. 7) demonstrates their coincidence. As the amplitude of the function $r(t)$ is very small in comparison to the equilibrium length $r_{0}$ the period of oscillation of the function $\varphi(t)$ doesn't change noticeably and is equal to the period of mathematical pendulum of the length $r_{0}$, namely, $T=2 \pi \sqrt{r_{0} / g}$ (see Eq. (4.8)). Obviously, period of the function $r(t)$ oscillation is two times less (see Eq. (4.10)).

If initial angular velocity $\dot{\varphi}_{0}$ is small enough and the corresponding amplitude $\varphi_{0}<\sqrt{4(\mu-1)}$ then the gravity force $m_{2} g$ is greater than the averaged tension $F_{t e n}^{a v}$ and the oscillating body $m_{1}$ will start to move up and the length of the pendulum $r$ will decrease. As the derivative $\dot{r}$ is negative in this case the term $2 \dot{r} \dot{\varphi} / r$ in the right-hand side of Eq. (4.2) will increase the amplitude of oscillation. When the amplitude becomes quite large the averaged tension exceeds the gravity force $m_{2} g$ and the acceleration $\ddot{r}$ changes its sign. As a result the bodies will stop and then start to move in opposite direction and the system will demonstrate quasi-periodic motion (see Fig. 4).

In case of greater angular velocity $\dot{\varphi}_{0}$ when $\varphi_{0}>\sqrt{4(\mu-1)}$ the averaged tension $F_{t e n}^{a v}$ is greater that the gravity force $m_{2} g$ and the body $m_{1}$ starts to move down first. Then the derivative $\dot{r}$ is positive and the term $2 \dot{r} \dot{\varphi} / r$ in the right-hand side of Eq. (4.2) acts like damping and decreases the amplitude of oscillation. When the amplitude will become small enough the gravity force $m_{2} g$ exceeds the averaged tension. As a result the bodies will stop and then start to move in opposite direction, and the system will demonstrate quasi-periodic motion again (see Fig. 8). 


\section{Conclusion}

In the present talk we have analyzed an influence of oscillation on the Atwood machine motion in the simplest case when only one body is permitted to oscillate in a plane. We have shown that even such oscillation can completely modify a motion of the system, while the simple Atwood machine demonstrates only the uniformly accelerated motion of the bodies. Of course, a mass and size of the pulley and changing the length $(r+R \varphi)$ between the body $m_{1}$ and pulley owing to winding the thread on the pulley affect on the system motion, as well. Doing necessary calculation, we have shown that these factors only change an inertness of the system and modify oscillation of the body $m_{1}$ but cannot change qualitatively the system behaviour.

It should be noted that there are many physical problems which seem to be quite simple although the corresponding mathematical models are rather complicated to be solved and analyzed by hand. But application of the modern computer algebra systems such as Wolfram Mathematica, for example, helps a lot in analyzing such problems and promotes development of physical intuition and better understanding of the subject.

Acknowledgements Many thanks to prof. Haiduke Sarafian for interesting discussion of the problem and useful remarks.

Open Access This article is distributed under the terms of the Creative Commons Attribution 4.0 International License (http:// creativecommons.org/licenses/by/4.0/), which permits unrestricted use, distribution, and reproduction in any medium, provided you give appropriate credit to the original author(s) and the source, provide a link to the Creative Commons license, and indicate if changes were made.

\section{References}

1. Atwood, G.: A Treatisa on the Rectilinear Motion and Rotation of Bodies. Cambridge University Press, Cambridge (1784)

2. Tufillaro, N.B., Abbott, T.A., Griffiths, D.J.: Swinging Atwood's machine. Am. J. Phys. 52, 895-903 (1984)

3. Tufillaro, N.B.: Motions of a swinging Atwood's machine. J. Phys. 46, 1495-1500 (1985)

4. Tufillaro, N.B.: Integrable motion of a swinging Atwood's machine. Am. J. Phys. 54, 142-143 (1986)

5. Casasayas, J., Nunes, T.A., Tufillaro, N.B.: Swinging Atwood's machine: integrability and dynamics. J. Phys. 51, 1693-1702 (1990)

6. Yehia, H.M.: On the integrability of the motion of a heavy particle on a tilted cone and the swinging Atwood's machine. Mech. Res. Commun. 33(5), 711-716 (2006)

7. Pujol, O., Pérez, J.P., Ramis, J.P., Simo, C., Simon, S., Weil, J.A.: Swinging Atwood machine: experimental and numerical results, and a theoretical study. Phys. D 239(12), 1067-1081 (2010)

8. Zeleny, E.: Swinging Atwood's machine. http://demonstrations.wolfram.com/SwingingAtwoodsMachine/ (2013). March 13, 2013

9. Wolfram, S.: The Mathematica Book, 5th edn. Wolfram Media, Champaign (2003)

10. Goldstein, H., Poole, C., Safko, J.: Classical Mechanics, 3rd edn. Addison Wesley, Reading (2000) 